\title{
Next-Generation Optical Technologies for Illuminating Genetically Targeted Brain Circuits
}

\author{
Karl Deisseroth, ${ }^{1,2}$ Guoping Feng, ${ }^{5}$ Ania K. Majewska, ${ }^{6}$ Gero Miesenböck, ${ }^{7}$ Alice Ting, ${ }^{8}$ and Mark J. Schnitzer ${ }^{3,4}$ \\ Departments of ${ }^{1}$ Bioengineering, ${ }^{2}$ Psychiatry and Behavioral Sciences, ${ }^{3}$ Biological Sciences, and ${ }^{4}$ Applied Physics, Stanford University, Stanford, California \\ 94305, ${ }^{5}$ Department of Neurobiology, Duke University Medical Center, Durham, North Carolina 27708, ${ }^{6}$ Department of Neurobiology and Anatomy, \\ University of Rochester, Rochester, New York 14627, ${ }^{7}$ Department of Cell Biology, Yale University School of Medicine, New Haven, Connecticut 06536, and \\ ${ }^{8}$ Department of Chemistry, Massachusetts Institute of Technology, Cambridge, Massachusetts 02139
}

Emerging technologies from optics, genetics, and bioengineering are being combined for studies of intact neural circuits. The rapid progression of such interdisciplinary "optogenetic" approaches has expanded capabilities for optical imaging and genetic targeting of specific cell types. Here we explore key recent advances that unite optical and genetic approaches, focusing on promising techniques that either allow novel studies of neural dynamics and behavior or provide fresh perspectives on classic model systems.

Optogenetic technology combines genetic targeting of specific neurons or proteins with optical technology for imaging or control of the targets within intact, living neural circuits. The roots of this approach extend back decades. The utility of labeling individual neurons was clear to Ramon y Cajal who conducted detailed histological analyses of single neurons embedded in dense neural circuitry using Golgi stains. The advent of combined optical/genetic methods now allows researchers to visualize genetically targeted neurons in living animals and to track and control electrical and biochemical events within targeted cell types.

The growth of optogenetic methodologies has included the advancement of several approaches: (1) one- and two-photon fluorescence microendoscopy for imaging fluorescently labeled cells deep within the mammalian brain; (2) simultaneous imaging of mammalian functional brain maps and the dynamics of neuronal morphology in genetically targeted neocortical cells; (3) genetically targeted reporters for imaging subcellular biochemical function in living neurons; (4) mouse transgenic techniques for genetic control of biochemical function in fluorescently labeled neurons embedded within intact mammalian circuitry; (5) genetically targeted optical control of neural activity in behaving Drosophila; and (6) high-speed, genetically targeted optical control of electrical activity in mammalian circuits. In this mini-

\footnotetext{
Received Sept. 5, 2006; revised Sept. 24, 2006; accepted Sept. 25, 2006.

This work was supported by the following: National Institute of Mental Health (NIMH), National Institute on Drug Abuse (NIDA), National Institute of General Medical Sciences (NIGMS), National Alliance for Research on Schizophrenia and Depression, American Psychiatric Institute for Research and Education, and the Snyder, Culpeper, Coulter Klingenstein, Whitehall, McKnight, and Albert Yu and Mary Bechmann Foundations (K.D.); NIMH and the Beckman and Ruth K. Broad Foundations (G.F.); the Burroughs-Wellcome fund (A.K.M.); NIH and Office of Naval Research (ONR) (G.M.); NIGMS and the McKnight and EJLB Foundations (A.T.); and NIDA, National Institute of Neurological Disorders and Stroke, National Institute on Deafness and Other Communication Disorders, National Science Foundation, ONR, the Beckman, Coulter, Klingenstein, Packard, and Sloan Foundations, and Mauna Kea Technologies (M.J.S.).

Correspondence should be addressed to either Karl Deisseroth or Mark J. Schnitzer. E-mail: deissero@stanford.edu or mschnitz@stanford.edu.

DOI:10.1523/JNEUROSCI.3863-06.2006

Copyright $\odot 2006$ Society for Neuroscience $\quad$ 0270-6474/06/2610380-07\$15.00/0
}

review, we highlight advances in these six areas and explore potential future applications of such technologies.

One- and two-photon fluorescence microendoscopy for chronic and portable cellular level imaging deep within the mammalian brain

Fluorescence microendoscopy is an emerging minimally invasive technology that allows cellular level imaging deep below the brain surface (Mehta et al., 2004; Flusberg et al., 2005b). Based on microlenses that are $350-1000 \mu \mathrm{m}$ in diameter and offer micrometer-scale optical resolution (Fig. 1A), one- and twophoton fluorescence versions of microendoscopy have enabled visualization of cells in mammalian brain areas that were previously inaccessible to in vivo imaging (Jung and Schnitzer, 2003; Jung et al., 2004; Levene et al., 2004). For example, the cochlea, which had long been impermeable to cellular level in vivo imaging because of its extreme delicacy and deep location in the temporal bone, can now be inspected by microendoscopy (Monfared et al., 2006), allowing visualization of individual auditory hair cells (Cheung et al., 2006). Generally, the two forms of microendoscopy provide complementary imaging data. The reliance on fluorescent markers, which often can be targeted genetically to specific cell types, implies that both varieties of microendoscopy are well suited for studies of neural circuit function.

Two-photon microendoscopy offers advantages similar to those of two-photon microscopy, including inherent optical sectioning and optical penetration through hundreds of micrometers of tissue, but uses a minimally invasive endoscope probe that can be inserted into deep tissues (Jung and Schnitzer, 2003; Jung et al., 2004; Levene et al., 2004). As in microscopy, two-photon excitation typically involves scanning an ultrashort-pulsed laser beam across the specimen, which has limited the maximal imaging frame rate obtained in vivo to tens of hertz at best. Although laser line-scanning imaging rates can reach the kilohertz range, by comparison, one-photon microendoscopy does not require laser illumination, allows images to be viewed by eye or on a camera, and permits frame rates of $>100 \mathrm{~Hz}$. Such capability appears 


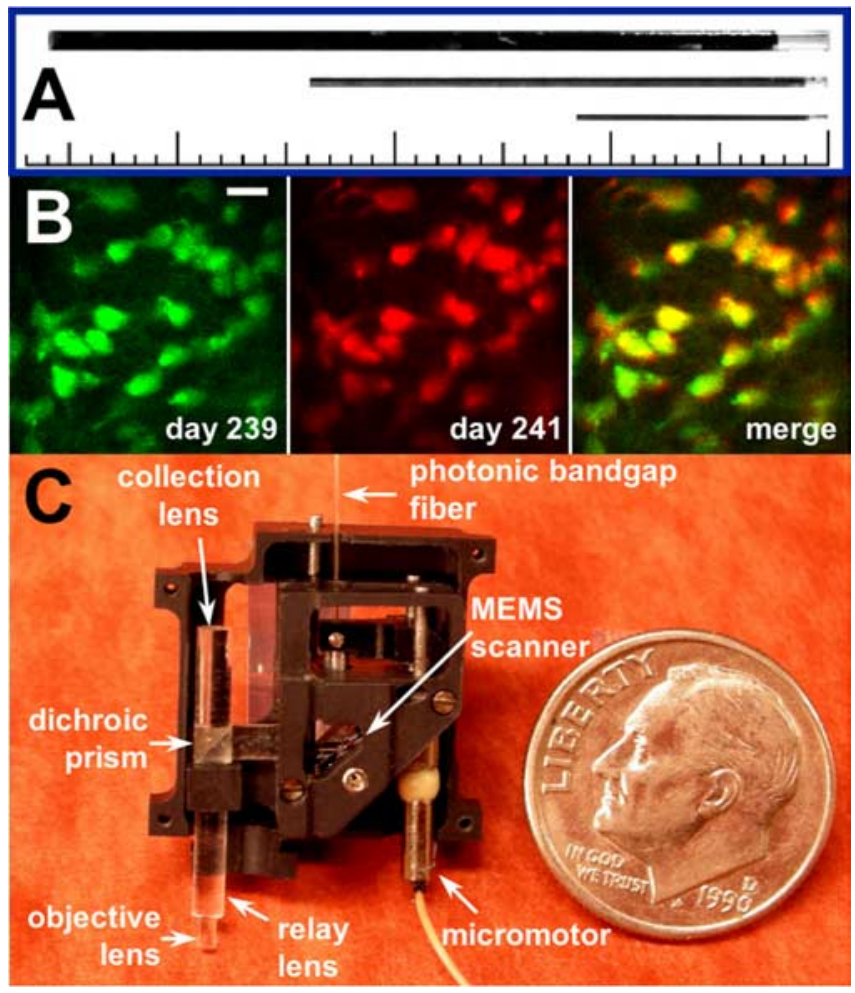

Figure 1. Chronic and portable fluorescence microendoscopy. A, Photograph of three microendoscope probes, which are 1000, 500, and $350 \mu \mathrm{m}$ in diameter. Each probe is a compound doublet gradient refractive index lens, comprising an endoscopic objective lens and a longer but weaker relay lens. The objective lenses are oriented to the right, and the relay lenses exhibit a dark coating. Minor ticks on the scale bar are $1 \mathrm{~mm}$ apart. Based on Jung et al. (2004). B, Images of CA1 hippocampal pyramidal cell bodies and proximal dendrites expressing YFP, acquired by two-photon microendoscopy in a live mouse. The mouse was of the YFP-H line, which expresses YFP under the control of the Thy1 promotor (Feng et al., 2000). On day 0, a guide tube was surgically implanted so that the microendoscope probe could be inserted repeatedly to the same tissue location just dorsal to CA1. The image acquired on day 239 after the initial surgery (left image, green pseudocolor) closely resembles that acquired on day 241 (middle image, red pseudocolor) as shown by the yellow portions in the merge of the two (right image). This indicates that the same cells have been visualized in each case. Scale bar, $10 \mu \mathrm{m}$. Data were taken from Jung et al. (2006). C, Photograph of the inner components of a portable fiber-optic two-photon microendoscopy device that is based on a microelectromechanical systems (MEMS) scanning mirror. A United States dime is shown for size comparison. The scanning mirror is microfabricated in silicon by photolithography methods and deflects light in two angular dimensions with a fast-axis scanning rate of $1.7 \mathrm{kHz}$ (Piyawattanametha et al., 2006). Ultrashort pulses of light from a titanium:sapphire laser are delivered to the instrument by a hollow-core photonic band-gap fiber that virtually eliminates ultrashort pulse distortion (Gobel et al., 2004a). The illumination deflects off the microelectromechanical systems mirror, which scans the light in a raster pattern. The light then enters an optical assembly consisting of a dichroic microprism and four microlenses. Two-photon excited fluorescence is generated in the sample at the focal spot of the objective lens, passes back undeflected through the microprism, and is focused by a collection lens into a multimode optical fiber (not shown) that routes to a photodetector. This portable imaging system was designed by Flusberg et al. (2006). Two-photon imaging using the microelectromechanical systems scanning mirror is described by Piyawattanametha et al. (2006).

suited for capturing rapid multi-cellular dynamics in vivo. A main limitation of one-photon microendoscopy, as in conventional epifluorescence microscopy, is the lack of optical sectioning, which reduces robustness to light scattering and thus limits optical penetration depths. Key goals of ongoing research on both microendoscopy modalities are to develop long-term and portable forms of imaging.

For studies of the peripheral nervous system and surface neocortical areas, chronic imaging preparations have been crucial for the iterative examination of cellular changes during development, disease progression, or plastic adaptation. A recently developed chronic preparation now allows repeated microendoscopy imaging of the mouse CA1 hippocampal area for durations that can exceed 1 year (Jung et al., 2006). A surgically implanted guide tube allows repetitive insertion of a microendoscope probe and thus the visualization over time of individual structures, such as genetically targeted cells expressing fluorescent proteins (Fig. $1 B)$. This approach should be useful for long-term studies of the spatiotemporal patterns of gene or protein expression. Applications include the study of animal disease models, which often involve genetically targeted expression of fluorescent markers within cell types implicated in the disease. Guide tubes can be implanted at multiple locations to provide imaging data from control and experimental sites to control for nonspecific effects of the imaging procedures. For example, in a long-term microendoscopy study of experimental glioma, imaging in one brain hemisphere provided control data from normal tissue, whereas imaging in the other hemisphere allowed repeated examination of deep brain tumor growth (Jung et al., 2006).

Fiber optic imaging technology offers the possibility of imaging cells in awake behaving animals (Helmchen et al., 2001). Toward this goal, portable fluorescence microendoscopy is based on flexible optical fibers that deliver light to and from the brain, allowing the light source and photodetectors to be physically separated from the imaging optics. One compact and lightweight (3.9 g) fiber-optic two-photon microendoscope for portable brain imaging relies on a single optical fiber for light delivery and another for fluorescence collection, allowing in vivo imaging with $\sim 1.2 \mu \mathrm{m}$ lateral resolution (Flusberg et al., 2005a). Another portable two-photon microendoscopy device uses a fiber optic bundle, which reduces image resolution but facilitates size reduction of the imaging head (Gobel et al., 2004b). Recently, a two-photon imaging system has been designed based on a microfabricated scanning mirror (Piyawattanametha et al., 2006) and is approximately the size of a dime (Fig. $1 C$ ). This device uses a focusing mechanism based on a direct current micromotor and has a mass of only $\sim 2.9$ g, sufficiently light to be borne by a freely moving adult mouse. Although considerable engineering work remains to make the forerunner devices described here user friendly, with further development, such technology seems well poised to allow studies of genetically identified cell types in behaving subjects. In the long run, the combination of chronic and portable microendoscopy techniques should be a potent dual approach for dissection of how neural circuit function evolves over time.

\section{Imaging circuit plasticity linked to genetically targeted neurons in vivo}

A distinct set of methods has arisen for simultaneous in vivo imaging of both neocortical functional brain maps and the structural dynamics of genetically targeted neurons. Such techniques are critical toward understanding how the brain remodels itself in response to environmental stimuli. Novel optical methods allow concurrent visualization of the dynamics of microscopic structures, such as synapses, and of global organizational properties, such as functional brain maps, in live mammalian subjects. These combined approaches have been applied to the study of ocular dominance plasticity.

By closing one eye during a well defined critical period, binocular neurons in visual cortex, which usually respond to stimulation in both eyes, can become driven predominantly by the open eye (Wiesel and Hubel, 1965). It is believed that initial changes in synaptic function are followed by slower alterations to 
neuronal structure that consolidate the rapid functional changes. Although physiological shifts in visual responses can be rapid after deprivation, occurring in hours to days, anatomical correlates such as the remodeling of axonal arbors can be relatively slow, occurring over weeks (Bent and Levelt, 2005). However, are structural changes really only a slow mechanism?

Novel in vivo imaging techniques used in combination with genetic labeling allow the determination of whether rapid changes in dendritic spine structure (Yuste and Bonhoeffer, 2001; Nimchinsky et al., 2002; Hayashi and Majewska, 2005) accompany early phases of visual plasticity. This approach relies on time-lapse two-photon microscopy of visual area V1 neurons in animals after lid suture and allows quantification of ongoing structural changes. In one set of imaging studies, the use of transgenic mice expressing green fluorescent protein (GFP) in a subset of pyramidal neurons in visual cortex [resembling the labeling pattern attained with a Golgi stain (Feng et al., 2000)] revealed that synapses were destabilized structurally not only with prolonged eye closure (Majewska and Sur, 2003) but also within $2 \mathrm{~d}$ of monocular deprivation (Oray et al., 2004). Dendritic spines in deprived animals exhibited significantly more growth, retraction, shape changes, and displacements over a $2 \mathrm{~h}$ imaging window. These studies demonstrate that dynamic changes in neuronal structure can occur rapidly after visual manipulations.

A follow-up study of the effects of visual deprivation tracked the dynamics of individual synapses in neurons with identified response properties in the ferret visual cortex. Injection of either herpes simplex (Neve et al., 2005) or attenuated Sindbis virus (Jeromin et al., 2003) expressing GFP into neocortical layer 2/3 enabled chronic two-photon imaging of fluorescently labeled neurons in the ferret preparation. In vivo intrinsic signal optical imaging was used to assign ocular dominance specificity to neurons spatially located in neocortical functional domains. Combining these imaging techniques with genetic labeling of individual cells revealed that neurons driven strongly by the deprived eye show extensive loss of dendritic spines within hours of the start of deprivation. Neurons that respond mostly to the open eye exhibit only moderate spine loss during this period (Yu et al., 2005). Thus, the joint application of genetic targeting with intrinsic signal and two-photon optical imaging yields a remarkably powerful system for the study of neural plasticity in the living brain.

\section{Genetically encoded reporters for imaging subcellular biochemical function}

An important new dimension to the optogenetic approach is optical imaging of individual biochemical events within genetically targeted neurons. A range of new methods have been developed for targeting probes (including fluorophores, photoaffinity labels, and quantum dots) to specific proteins in living neurons (Chen et al., 2005). The common feature of all of these methods is that they only require the protein of interest to be fused to a short peptide sequence. For example, to label the AMPA receptor with quantum dots, the receptor can be fused to a 15 amino acid "acceptor peptide" (AP), a substrate for biotin ligase (Howarth et al., 2005, 2006). The AP-tagged receptor can then be expressed in neurons and biotinylated at the extracellular side by adding biotin ligase and biotin-AMP ester to the medium for $1 \mathrm{~min}$. The ligated biotin then can be recognized using streptavidin-quantum dot conjugates. The total labeling time is only $2 \mathrm{~min}$, and the specificity and speed of labeling can be excellent. Indeed, because of the intense brightness and the virtual lack of photobleaching of quantum dots, it is possible to image the trafficking of single molecules of AMPA receptors in real time (Howarth et al., 2005,
2006). This novel labeling approach allows selective visualization of cell surface, and thus physiologically active, receptor molecules in living neurons.

A related two-step labeling protocol can be used to tag proteins but avoid the steric bulk of streptavidin. Biotin ligase can use analogs of biotin such as a ketone isostere (Chen et al., 2005). After ligating the ketone specifically to AP-fusion proteins expressed on the surface of live cells, the ketone can be derivatized for non-streptavidin-based tagging using hydrazide-functionalized probes. Other ligase enzymes also can be used, including transglutaminase (Lin and Ting, 2006) and lipoic acid ligase (LplA); the latter, from E. coli, accepts alkyl azide probes in place of lipoic acid. Because cells do not naturally possess alkyl azides, and azides can be selectively modified with cyclooctyne-functionalized probes, LplA is potentially useful for labeling intracellular protein targets in which the biotin ligase methods would not be suitable because of endogenous ketones and aldehydes. Indeed, a combination of these orthogonal labeling schemes can be used to image several different neuronal proteins in the same living cell. For example, these new labeling methods could be used simultaneously to study protein trafficking during new synapse formation, identify the order in which presynaptic and postsynaptic proteins arrive at nascent synaptic sites, and determine how different isoforms of synaptic proteins are selectively targeted to excitatory or inhibitory synapses.

\section{Genetic manipulation and imaging of single neurons within intact circuits}

An optogenetic approach complementary to imaging biochemical function would be controlling biochemical function in genetically targeted neurons that express fluorescent markers. Recently, a powerful labeling approach that achieves this goal has been developed. It allows genetic manipulations to be induced in a labeled population of neurons that is embedded within otherwise wild-type tissue. In short, this approach resembles a functional genetic version of the Golgi stain.

Many lines of transgenic mice have been generated in which GFP labels small subsets of neurons, allowing cell-by-cell tracking of processes such as axonal guidance, dendritic growth, and synaptic dynamics. This effect was achieved by taking advantage of a strong neuron-specific promoter (Thy1) and a transgenic phenomenon called "position effect variegation" that gives rise to neuronal labeling with varying degrees of sparseness in different lines of transgenic mice (Feng et al., 2000; Young and Feng, 2004). Now, a functional-genetic component has been added by expressing CreER ${ }^{\mathrm{T} 2}$, a tamoxifen-inducible form of the Cre recombinase, in the same cells as the fluorescent protein using two copies of the Thyl promoter (Fig. 2 A). The Thyl-yellow fluorescent protein (YFP)/CreER approach thus combines the powerful cre/loxP system for performing conditional gene knock-out or expression and the Golgi-like fluorescent labeling of neurons to reveal their morphology (Fig. 2B,C). Genetic manipulation is spatially restricted to small subsets of labeled neurons and can be temporally controlled through administration of the activating drug tamoxifen. Accordingly, these animals have been named "SLICK" mice (for single-neuron labeling with inducible Cremediated knock-out). By crossing SLICK mice to a line of Cre reporter mice in which the expression of LacZ depends on Cremediated excision of a loxP-flanked transcription stop cassette, administration of tamoxifen can be shown to efficiently induce LacZ expression only in YFP-positive neurons (Fig. 2). In mouse lines in which labeling is sparse and labeled neurons are far apart, genetic manipulation and visualization at the single-neuron level is possible. 

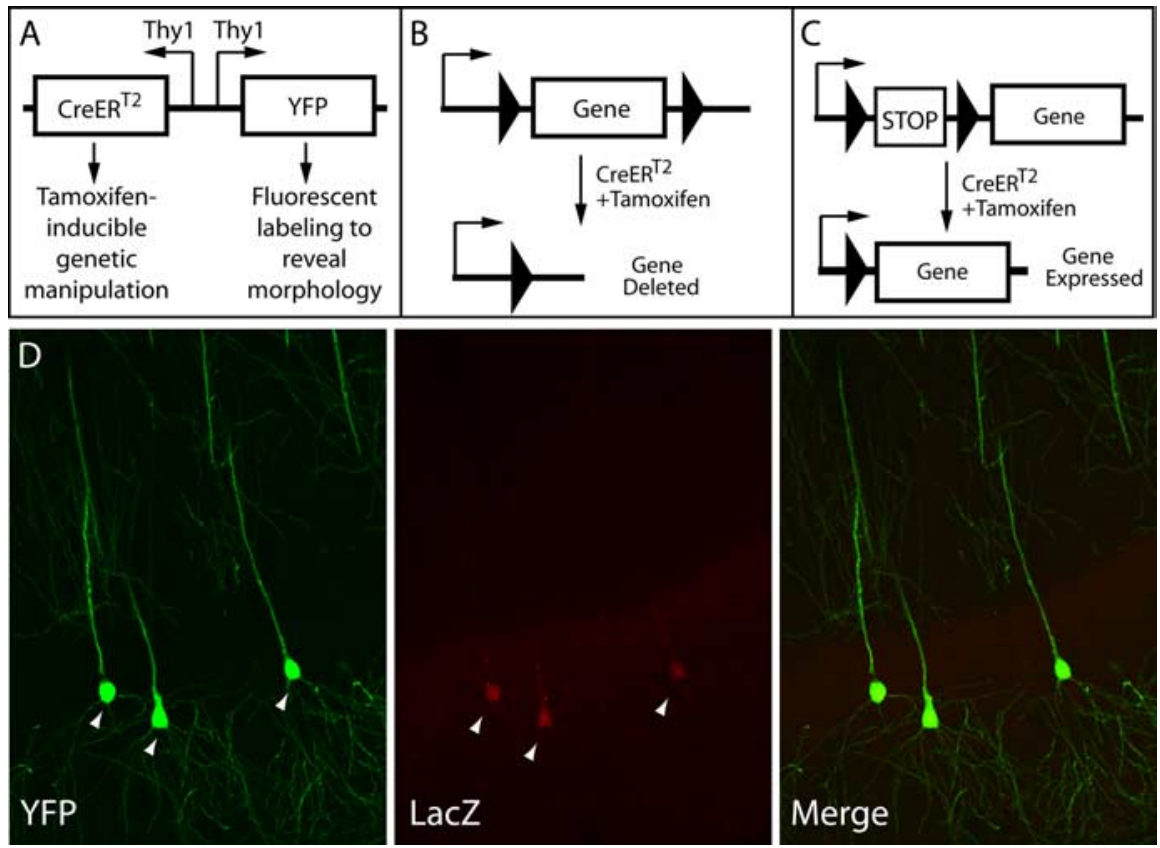

Figure 2. Schematic description of SLICK mice. $\boldsymbol{A}$, The construct used to generate SLICK transgenic mice consists of two copies of the Thy1 promoter placed back to back. One copy of Thy1 drives expression of a fluorescent protein (YFP), whereas the other expresses an inducible form of (re recombinase $\left(\mathrm{CreER}^{\mathrm{T} 2}\right)$. $\boldsymbol{B}$, Gene ablation with SLICK mice. Cre recombinase can delete DNA sequences located between loxP recognition sites (black triangles) and can therefore be used for conditional deletion of genes. $\boldsymbol{C}$, Induction of transgene expression by SLICK mice. To generate an inducible line of transgenic mice, a transcriptional stop cassette (STOP) is flanked by loxP sites and placed in front of the transgene. When activated, Cre recombinase in SLICK mice can delete the STOP cassette and induce transgene expression. D, Tamoxifen-induced CreER-mediated recombination in SLICK mice. SLICK mice were crossed to a line of Cre reporter mice, Rosa26-loxP-STOP-IoxP-LacZ, in which the lacZ expression depends on Cre-mediated excision of transcriptional STOP cassette. When the SLICK/Rosa26-I0xP-STOP-loxP-LacZ mice were treated with tamoxifen, which activates CreER, the expression of LacZ is efficiently induced in YFP-labeled neurons (arrowheads).

The ability to manipulate labeled neurons genetically in such a precise manner has several advantages. First, Golgi-like fluorescent labeling permits the analysis of detailed morphology and synaptic connections of individual mutant neurons. Second, the restriction of genetic manipulation to a small subset of labeled neurons allows analysis to be performed in a predominantly wild-type background, thereby avoiding large-scale disruption of neural circuits and allowing cell-autonomous functions of genes to be distinguished from indirect effects of widespread gene deletion. Third, the inducible nature of genetic manipulation with the SLICK system permits analysis of gene function at late developmental or adult stages, thereby avoiding the potential complications of embryonic lethality, early developmental defects or compensation for a deleted gene. Finally, the YFP labeling of neurons in the SLICK mice is constitutive. This allows labeled wild-type neurons from untreated animals to be used as controls for genetically manipulated cells. With in vivo imaging of YFPlabeled neurons in accessible areas of the nervous system, it should be possible to image cells before and after a genetic manipulation, thereby examining neuronal properties in the presence and absence of a given gene in the same neuron in vivo.

\section{Genetically targeted optical control of neural activity} and behavior

It is now possible to control events in targeted neurons not only genetically but also optically. Over the past several years, several approaches for optical control of neuronal activity have been developed (Zemelman et al., 2002, 2003; Banghart et al., 2004; Boyden et al., 2005; Lima and Miesenböck, 2005; Schroll et al.,
2006; Volgraf et al., 2006). Among the more exciting uses for this technology is probing the neuronal substrates of behavior (Lima and Miesenböck, 2005; Nagel et al., 2005). The capacity to control remotely, simply by illumination, genetically delineated sets of neuronal targets has opened experimental avenues that were inaccessible using conventional microelectrode approaches. These include simultaneous control of large numbers of neurons in arbitrary anatomical arrangements, control of neuronal targets without advance knowledge of their spatial locations, selective control of neurons with defined functional identities, and stable control of neuronal targets whose positions change unpredictably in freely moving animals.

Major strengths of this approach, namely non-invasiveness, parallelism, cell-type selectivity, and tolerance for motion, are illustrated in a study of how dopamine regulates movements in fruit flies (Lima and Miesenböck, 2005). As in vertebrates, only a minority $(\sim 0.1 \%)$ of all neurons in the CNS of Drosophila synthesize dopamine. The 150 or so dopaminergic neurons are distributed among several neuronal clusters that are dispersed throughout the brain. Consider what it would take to manipulate these neurons by electrophysiological techniques. Even if one knew the identity and location of each cell (which is generally not the case) and even if the scale of the CNS permitted facile electrode placement (which also is not the case), handling 150 electrodes simultaneously is prohibitively difficult. Moreover, even if it were possible to overcome this technical difficulty, an animal immobilized on a bed of microelectrodes could no longer display the behavior of interest, thus defeating the very purpose of the experiment.

The dopaminergic neurons, however, now can be manipulated selectively with light. Under control of the tyrosine hydroxylase gene promoter, a heterologous ligand-gated cation channel not present in the fly was expressed in dopaminergic neurons (Lima and Miesenböck, 2005). Flies were injected with a biologically inert ligand sequestered in a photolabile chemical "cage." Brief illumination of the freely moving animals released free ligand, which in turn activated only those neurons that had been programmed genetically to express the receptor. Optical activation of dopaminergic neurons elicited two behavioral changes: a state-dependent increase or decrease in global locomotor activity, and a shift of the flies' navigation trajectories from centrophobic to centripetal movement patterns. Changes in the animals' preference for the open field or the sheltered periphery may be caused by artificially altered expectations of reward or punishment, a notion that ongoing experiments continue to explore.

In addition to establishing causal connections between the activities of specific sets of neurons and the expression of particular behaviors, general principles of neural control are beginning to emerge from these behavioral experiments. Although the actions of dopamine illustrate a layer of neuromodulatory control of movement, many fundamental aspects of insect locomotion, 
such as transitions between movement and rest, or between different forms of movement, appear to be regulated by a handful of so-called command neuron systems. Genetically targeted photostimulation of one such system, the command neurons dedicated to escape behaviors (Lima and Miesenböck, 2005) has demonstrated a general approach to defining the physiological roles of these circuits (Miesenböck and Kevrekidis, 2005).

Millisecond-scale genetically targeted optical control of intact mammalian neural circuits

For experiments in mammals as well as in the invertebrate preparations, a new technology allows optical control with high temporal resolution and may permit the analysis of mammalian neural codes on the millisecond timescale. This novel highspeed method for optical control of neurons (Boyden et al., 2005) uses a gene derived from the alga Chlamydomonas reinhardtii that encodes a light-activated cation channel [channelrhodopsin-2 (ChR2)] (Nagel et al., 2003; Hegemann et al., 2005). Introduction of engineered ChR2-XFP (fluorescent protein) fusions into isolated neurons (Boyden et al., 2005) or hippocampal circuits (Fig. 3) (Zhang et al., 2006), followed by delivery of millisecondscale pulses of blue light, results in the unprecedented capability to drive consistent, sustained trains of light-triggered activity with single-action potential resolution, in mammalian neural tissue (Zhang et al., 2006). Among the important properties of this technology are highly precise spike timing ( $<5 \mathrm{~ms}$ jitter) and highly reproducible spike firing $(>98 \%)$ even in long trains (Boyden et al., 2005; Zhang et al., 2006). In addition, ChR2-mediated millisecond control of action potentials also can be used to control synaptic activity with high precision (Fig. 3). By stereotaxically delivering ChR2enhanced YFP (EYFP) lentiviral vectors into the dentate gyrus of the hippocampal formation, it is possible to express ChR2-EYFP in a large fraction of granule cells (Fig. 3). Simultaneous excitation of ChR2-positive cells with blue light and patch recording in ChR2-negative pyramidal cells along the mossy fiber pathway in CA3 (Fig. 3A) show clear light-evoked excitatory transmission in the ChR2-negative cell (Fig. $3 B-D)$. By turning down the duration of light pulses, it is also possible to reduce the magnitude of light-evoked postsynaptic currents (Fig. 3E), likely via recruitment of fewer ChR2-expressing axons (as can be done with a stimulating electrode by turning down the current). Using ChR2 in this way opens the door to a wide range of questions focusing on the synaptic influence of specific genetically defined classes of cells in intact circuitry, a long-sought goal of synaptic physiologists and systems neuroscientists.

A

B

E
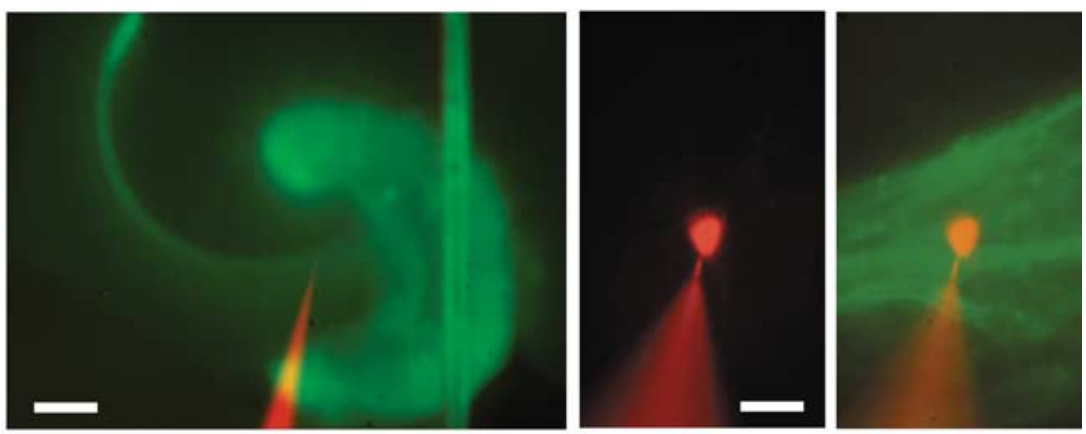

C

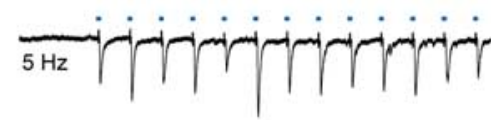

+ CNQX

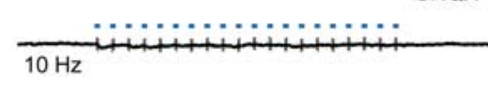

CNQX washout
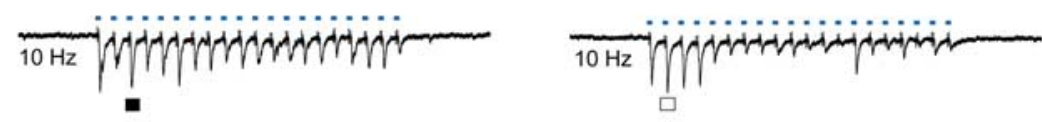

D
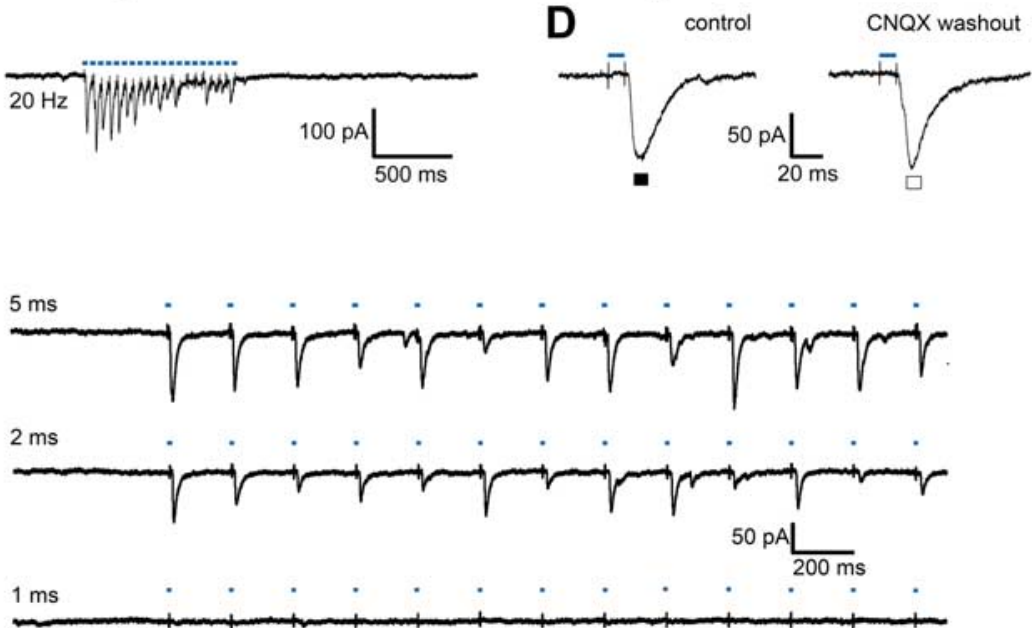

Figure 3. Precisely timed, light-driven synaptic transmission within intact mammalian brain tissue. $\boldsymbol{A}$, Left, ChR2-EYFP expression in an acute hippocampal slice. Data courtesy of Zhang et al. (2006) and Wang et al. (2006). The recording pipette shown is filled with an intracellular solution containing Alexa Fluor $594(10 \mu \mathrm{g} / \mathrm{ml})$. Middle, The recorded ChR2-EYFP-negative cell filled with Alexa Fluor 594. Right, Combined image of the recorded cell (red) and mossy fiber projections (green). Scale bars: left, 200 $\mu \mathrm{m}$; middle and right, $20 \mu \mathrm{m}$. B, Excitatory synaptic transmission driven by 5,10 , and $20 \mathrm{~Hz}$ repeated light pulses (each blue dash represents one $15 \mathrm{~ms}$ light flash). $\boldsymbol{C}$, The selective glutamatergic transmission blocker CNQX (20 $\mu \mathrm{m})$ abolishes the synaptic response. Calibration is the same as in $\boldsymbol{B}$. D, A single postsynaptic event before (filled square in $\boldsymbol{B}$ ) and after (open square in $\boldsymbol{C}$ (NNQX application in expanded timescale. $\boldsymbol{E}$, The $10 \mathrm{~Hz}$ synaptic responses evoked via 5, 2, and $1 \mathrm{~ms}$ light flashes. All traces were collected without the addition of exogenous all-trans-retinal. This cofactor independence and the demonstrated temporal precision offer considerable utility for other domains of neuroscience and neuroengineering, with potential applications ranging from stem cell engineering (Stroh et al., 2006), to high-throughput optical screening of drugs, to high-speed optics-based neural interfaces for nervous tissue repair (Bi et al., 2006).

Importantly, in mammals, no added cofactors are needed and none were added at any point the experiments shown in Figure 3. Photons are absorbed by the all-trans-retinal cofactor of ChR2 that is endogenously present at sufficient levels in vertebrate CNS tissue (Fig. 3) (Li et al., 2005; Bi et al., 2006; Ishizuka et al., 2006; Zhang et al., 2006). Therefore, this technology is more suitable for use within intact living large animals such as mammals than uncaging-based methods that require the addition of large quantities of custom cofactors. As noted above, this limitation of uncaging methods does not extend to small animals such as Drosophila. Because crucial aspects of mammalian neural circuitry operate on the millisecond timescale, this optogenetic method 
also is suitable for probing specific high-speed electrical activity patterns in neural circuit dynamics and behavior.

\section{Summary}

One can only imagine that the early cellular neuroanatomists might have been surprised and pleased to see their work extended by optogenetic approaches. The power of labeling individual neurons within intact tissue has been expanded by techniques for long-term in vivo imaging as well as for biochemical and electrical control of genetically targeted neurons within intact living neural circuits. These methods may prove to be especially useful in combination. It will be particularly intriguing to combine genetically targeted photostimulation or biochemical-control methods (see above, sections 4-6) with the emerging fluorescence imaging modalities (see above, sections 1-3) in vivo. Although Ramon y Cajal's conclusions about neural function were attained in an essentially inferential manner, the modern combination of optical techniques may lead to a more complete toolkit for performing causal studies on the roles of specific genes and cells within functioning neural circuitry. Thus, joint uses of light to image and control identified circuits will contribute to a greater synthetic understanding of the principles underlying neural computation.

\section{References}

Banghart M, Borges K, Isacoff E, Trauner D, Kramer RH (2004) Lightactivated ion channels for remote control of neuronal firing. Nat Neurosci 7:1381-1386.

Bent M, Levelt C (2005) Structural plasticity in the developing visual system. Prog Brain Res 147:125-139.

Bi A, Cui J, Ma YP, Olshevskaya E, Pu M, Dizhoor AM, Pan ZH (2006) Ectopic expression of a microbial-type rhodopsin restores visual responses in mice with photoreceptor degeneration. Neuron 50:23-33.

Boyden ES, Zhang F, Bamberg E, Nagel G, Deisseroth K (2005) Millisecondtimescale, genetically targeted optical control of neural activity. Nat Neurosci 8:1263-1268.

Chen I, Howarth M, Lin W, Ting AY (2005) Site-specific labeling of cell surface proteins with biophysical probes using biotin ligase. Nat Methods 2:99-104.

Cheung EL, Monfared A, Popelka G, Blevins NH, Schnitzer MJ (2006) In vivo assessment of mammalian auditory hair cell functionality using fluorescence microendoscopy. Soc Neurosci Abstr 32:45.6.

Feng G, Mellor RH, Bernstein M, Keller-Peck C, Nguyen QT, Wallace M, Nerbonne JM, Lichtman JW, Sanes JR (2000) Imaging neuronal subsets in transgenic mice expressing multiple spectral variants of GFP. Neuron 28:41-51.

Flusberg BA, Jung JC, Cocker ED, Anderson EP, Schnitzer MJ (2005a) In vivo brain imaging using a portable 3.9 gram two-photon fluorescence microendoscope. Opt Lett 30:2272-2274.

Flusberg BA, Cocker ED, Piyawattanametha W, Jung JC, Cheung EL, Schnitzer MJ (2005b) Fiber-optic fluorescence imaging. Nat Methods 2:941-950.

Flusberg BA, Cocker ED, Piyawattanametha W, Jung JC, Barretto RP, Ko TH, Ra H, Lee D, Solgaard O, Schnitzer MJ (2006) Portable forms of fiberoptic one- and two-photon fluorescence microendoscopy toward imaging in freely moving mice. Soc Neurosci Abstr 32:834.838.

Gobel W, Nimmerjahn A, Helmchen F (2004a) Distortion-free delivery of nanojoule femtosecond pulses from a Ti:sapphire laser through a hollowcore photonic crystal fiber. Opt Lett 29:1285-1287.

Gobel W, Kerr JN, Nimmerjahn A, Helmchen F (2004b) Miniaturized twophoton microscope based on a flexible coherent fiber bundle and a gradient-index lens objective. Opt Lett 29:2521-2523.

Hayashi Y, Majewska AK (2005) Dendritic spine geometry: functional implication and regulation. Neuron 46:529-532.

Hegemann P, Ehlenbeck S, Gradmann D (2005) Multiple photocycles of channelrhodopsin. Biophys J 89:3911-3918.

Helmchen F, Fee MS, Tank DW, Denk W (2001) A miniature headmounted two-photon microscope. high-resolution brain imaging in freely moving animals. Neuron 31:903-912.
Howarth M, Takao K, Hayashi Y, Ting AY (2005) Targeting quantum dots to surface proteins in living cells with biotin ligase. Proc Natl Acad Sci USA 102:7583-7588.

Howarth M, Chinnapen DJ, Gerrow K, Dorrestein PC, Grandy MR, Kelleher NL, El-Husseini A, Ting AY (2006) A monovalent streptavidin with a single femtomolar biotin binding site. Nat Methods 3:267-273.

Ishizuka T, Kakuda M, Araki R, Yawo H (2006) Kinetic evaluation of photosensitivity in genetically engineered neurons expressing green algae light-gated channels. Neurosci Res 54:85-94.

Jeromin A, Yuan LL, Frick A, Pfaffinger P, Johnston D (2003) A modified Sindbis vector for prolonged gene expression in neurons. J Neurophysiol 90:2741-2745.

Jung JC, Schnitzer MJ (2003) Multiphoton endoscopy. Opt Lett 28:902-904.

Jung JC, Mehta AD, Aksay E, Stepnoski R, Schnitzer MJ (2004) In vivo mammalian brain imaging using one- and two-photon fluorescence microendoscopy. J Neurophysiol 92:3121-3133.

Jung JC, Ko TH, Barretto RP, Waters A, Wang TJ, Capps G, Kim S, Recht L, Schnitzer MJ (2006) Long-term imaging of deep brain neurons, microcirculation, and tumor growth using fluorescence microendoscopy. Soc Neurosci Abstr 32:389.25.

Levene MJ, Dombeck DA, Kasischke KA, Molloy RP, Webb WW (2004) In vivo multiphoton microscopy of deep brain tissue. J Neurophysiol 91:1908-1912.

Li X, Gutierrez DV, Hanson MG, Han J, Mark MD, Chiel H, Hegemann P, Landmesser LT, Herlitze S (2005) Fast noninvasive activation and inhibition of neural and network activity by vertebrate rhodopsin and green algae channelrhodopsin. Proc Natl Acad Sci USA 102: $17816-17821$.

Lima SQ, Miesenböck G (2005) Remote control of behavior through genetically targeted photostimulation of neurons. Cell 121:141-152.

Lin CW, Ting AY (2006) Transglutaminase-catalyzed site-specific conjugation of small-molecule probes to proteins in vitro and on the surface of living cells. J Am Chem Soc 128:4542-4543.

Majewska A, Sur M (2003) Motility of dendritic spines in visual cortex in vivo: changes during the critical period and effects of visual deprivation. Proc Natl Acad Sci USA 100:16024-16029.

Mehta AD, Jung JC, Flusberg BA, Schnitzer MJ (2004) Fiber optic in vivo imaging in the mammalian nervous system. Curr Opin Neurobiol 14:617-628.

Miesenböck G, Kevrekidis IG (2005) Optical imaging and control of genetically designated neurons in functioning circuits. Annu Rev Neurosci 28:533-563.

Monfared A, Blevins NH, Cheung EL, Jung JC, Popelka G, Schnitzer MJ (2006) In vivo imaging of mammalian cochlear blood flow using fluorescence microendoscopy. Otol Neurotol 27:144-152.

Nagel G, Szellas T, Huhn W, Kateriya S, Adeishvili N, Berthold P, Ollig D, Hegemann P, Bamberg E (2003) Channelrhodopsin-2, a directly lightgated cation-selective membrane channel. Proc Natl Acad Sci USA 100:13940-13945.

Nagel G, Brauner M, Liewald JF, Adeishvili N, Bamberg E, Gottschalk A (2005) Light activation of channelrhodopsin-2 in excitable cells of Caenorhabditis elegans triggers rapid behavioral responses. Curr Biol 15:2279-2284.

Neve RL, Neve KA, Nestler EJ, Carlezon WA, Jr (2005) Use of herpes virus amplicon vectors to study brain disorders. Biotechniques 39:381-391.

Nimchinsky EA, Sabatini BL, Svoboda K (2002) Structure and function of dendritic spines. Annu Rev Physiol 64:313-353.

Oray S, Majewska A, Sur M (2004) Dendritic spine dynamics are regulated by monocular deprivation and extracellular matrix degradation. Neuron 44:1021-1030.

Piyawattanametha W, Barretto RP, Ko TH, Flusberg BA, Cocker ED, Ra H, Lee D, Solgaard O, Schnitzer MJ (2006) Fast-scanning two-photon fluorescence imaging based on a microelectromechanical systems twodimensional scanning mirror. Opt Lett 31:2018-2020.

Schroll C, Riemensperger T, Bucher D, Ehmer J, Voller T, Erbguth K, Gerber B, Hendel T, Nagel G, Buchner, Fiala A (2006) Light-induced activation of distinct modulatory neurons triggers appetitive or aversive learning in Drosophila larvae. Curr Biol 16:1741-1747. 
Stroh A, Wang L, Zhang F, Deisseroth K (2006) Neuronal differentiation of mouse embryonic stem cells expressing the light-sensitive ion channel Channelrhodopsin-2. Soc Neurosci Abstr 32:112.6.

Volgraf M, Gorostiza P, Numano R, Kramer RH, Isacoff EY, Trauner D (2006) Allosteric control of an ionotropic glutamate receptor with an optical switch. Nat Chem Biol 2:47-52.

Wang L, Zhang F, Deisseroth K (2006) Temporally precise, all-optical probing of neuronal activity in intact brain tissue. Soc Neurosci Abstr 32:98.4.

Wiesel TN, Hubel DH (1965) Comparison of the effects of unilateral and bilateral eye closure on cortical unit responses in kittens. J Neurophysiol 28:1029-1040.

Young P, Feng G (2004) Labeling neurons in vivo for morphological and functional studies. Curr Opin Neurobiol 14:642-646.
Yu H, Majewska A, Sur M (2005) Rearrangement of dendritic spines during short-term monocular deprivation in the primary visual cortex of the ferret in vivo. Soc Neurosci Abstr 31:714.8.

Yuste R, Bonhoeffer T (2001) Morphological changes in dendritic spines associated with long-term synaptic plasticity. Annu Rev Neurosci 24:1071-1089.

Zemelman BV, Lee GA, Ng M, Miesenböck G (2002) Selective photostimulation of genetically chARGed neurons. Neuron 33:15-22.

Zemelman BV, Nesnas N, Lee GA, Miesenböck G (2003) Photochemical gating of heterologous ion channels: remote control over genetically designated populations of neurons. Proc Natl Acad Sci USA 100:1352-1357.

Zhang F, Wang LP, Boyden ES, Deisseroth K (2006) Channelrhodopsin-2 and optical control of excitable cells. Nat Methods 3:785-792. 